\title{
A Characterization of the Dependence of the Riemannian Metric on the Curvature Tensor by Young Symmetrizers
}

\author{
B. Fiedler
}

\begin{abstract}
In differential geometry several differential equation systems are known which allow the determination of the Riemannian metric: from the curvature tensor in normal coordinates. We consider two of such differential equation systems. The first system used by Günther [8] yields a power series of the metric the coefficients of which depend on the covariant derivatives of the curvature tensor symmetrized in a certain manner. The second system, the so-called Herglotz relations [9], leads to a power series of the metric depending on symmetrized partial derivatives of the curvature tensor.

We determine a left ideal of the group ring $\mathbb{C}\left[\mathcal{S}_{r+4}\right]$ of the symmetric group $\mathcal{S}_{r+4}$ which is associated with the partial derivatives $\partial^{(\tau)} R$ of the curvature tensor $R$ of order $r$ and construct a decomposition of this left ideal into three minimal left ideals using Young symmetrizers and the Littlewood-Richardson rule. Exactly one of these minimal left ideals characterizes the so-called essential part of $\partial^{(r)} R$ on which the metric really depends via the Herglotz relations. We give examples of metrics with and without a non-essential part of $\partial^{(r)} R$. Applying our results to the covariant derivatives of the curvature tensor we can show that the algebra of tensor polynomials $\mathcal{R}$ generated by $\nabla_{\left(i_{1}\right.} \ldots \nabla_{\left.i_{r}\right)} R_{i j k l}$ and the algebra $\mathcal{R}^{s}$ generated by $\nabla_{\left(i_{1}\right.} \ldots \nabla_{i_{r}} R_{\left.|k| i_{r+1} i_{r+2}\right) l}$ fulfil $\mathcal{R}=R^{s}$.

Keywords: Calculation of a metric, curvalure tensor, partial derivatives of the curvature tensor, covariant derivatives of the curvature tensor, algebras of tensor polynomials, Herglotz relations, power series method, minimal left ideals, Young symmetrizers, Littleuood-Richordson rule, use of computer algebra systems.
\end{abstract}

AMS subject classification: Primary $05 \mathrm{E} 10,53 \mathrm{C} 21$, secondary $20 \mathrm{C} 05,53 \mathrm{~A} 55,53$ - 04

\section{Introduction}

Several investigations in differential geometry and general relativity theory make use of certain differential equation systems which allow to determine a pseudo-Riemannian metric from its Riemannian curvature tensor in normal coordinates. P. Günther has established the following construction of a differential equation system of such a type in [8: Appendix I].

Let $(M, g)$ be an $n$-dimensional analytic pseudo-Riemannian manifold with metric $g$ and Levi-Civita comnection $\nabla$, and let $\{U, x\}$ be a normal coordinate system of $(M, g)$

B. Fiedler: Universität Leipzig, Mathematisches Institut, Augustuspi 10/11, D-04109 Leipzig, Germany

ISSN 0232-2064/\$2.50 (C) Heldermam Verlag Berlin 
around the centre $P_{0} \in U \subseteq M$, i.e. $x\left(P_{0}\right)=0$. If we choose an orthonormal basis $\left\{v_{1}, \ldots, v_{n}\right\} \subset M_{P_{0}}$ of the tangent space $M_{P_{0}}$ of the manifold $M$ in the point $P_{0}$ and carry out a parallel transport of this basis along every geodesic starting in $P_{0}$, we obtain $n$ smooth vector fields $\left\{X_{1}, \ldots, X_{n}\right\}$ on a suitable open neighbourhood $U^{\prime} \subseteq U$ of $P_{0}$ which form an $n$-frame in every point of $U^{\prime}$. We denote by $T_{A_{1} \ldots A_{r}}:=T\left(X_{A_{1}}, \ldots, X_{A_{r}}\right)$ the coordinates of a covariant tensor field $T$ of order $r$ with respect to $\left\{X_{1}, \ldots, X_{n}\right\}$ and by $T_{i_{1} \ldots i_{r}}:=T\left(\partial_{i_{1}}, \ldots, \partial_{i_{r}}\right)$ the coordinates of the same tensor field with respect to the basis vector fields $\partial_{i}:=\partial / \partial x^{i}$ of the normal coordinate system $\{U, x\}$. Then there hold true the relations

$$
g_{i j}=\sigma_{i}^{A} \sigma_{j}^{B} g_{A B} \quad, \quad g_{A B}=\sigma_{A}^{i} \sigma_{B}^{j} g_{i j}
$$

with the transformation matrices ${ }^{1)} \sigma:=\left(\sigma_{i}^{A}\right)$ and $\sigma^{-1}:=\left(\sigma_{A}^{i}\right)$ defined by

$$
\partial_{i}=\sigma_{i}^{A} X_{A} \quad, \quad X_{A}=\sigma_{A}^{i} \partial_{i} .
$$

The coordinates $g_{A B}$ in (1.1) fulfil

$$
g_{A B}=\text { const }=\left\{\begin{array}{cll} 
\pm 1 & \text { if } & A=B \\
0 & \text { if } & A \neq B
\end{array}\right.
$$

where the number of +1 and -1 in (1.2) is given by the signature of the metric $g$.

P. Günther has shown in [8: Appendix I] that the matrix $\sigma$ satisfies on an open neighbourhood of $P_{0}$ the relation ${ }^{2)}$

$$
X X(\sigma)+X(\sigma)+\sigma \cdot Q=0 .
$$

Here $X$ denotes the vector field $X:=x^{i} \partial_{i}$ formed from the normal coordinates $x^{i}$. Further, $Q$ is an analytic $(n \times n)$-matrix-valued function with power series $Q=\sum_{l=2}^{\infty} Q_{(l)}$ the summands $Q_{(l)}$ of which are obtained by the equation $Q_{(l)}=\sigma^{-1}\left(P_{0}\right) \cdot R_{(l)} \cdot \sigma\left(P_{0}\right)$ from analytic $(n \times n)$-matrices $R_{(l)}$ which depend on the covariant derivatives of the Riemannian curvature tensor ${ }^{3)} R_{i j k l}$ according to

$$
\begin{aligned}
& R_{(2)}:=\left(R_{a i_{1} i_{2} b}\left(P_{0}\right) x^{i_{1}} x^{i_{2}}\right)_{a, b=1, \ldots, n} \\
& R_{(l)}:=\left(\frac{1}{(l-2) !}\left(\nabla_{i_{1}} \ldots \nabla_{u_{1-2}} R_{a i_{-1} i_{1} b}\right)\left(P_{0}\right) x^{i_{1}} \ldots x^{i_{l}}\right)_{a, b=1, \ldots, n} \quad, l \geq 3 .
\end{aligned}
$$

Often, investigations in differential geometry use the algebra

$$
\mathcal{R}:=\left\langle g_{\imath j} ; g^{i j} ; R_{i j k l} ; \nabla_{i_{1}} \ldots \nabla_{i_{r}} R_{i j k l}, r \geq 1\right\rangle
$$

1) The matrix $\left(\sigma_{A}^{i}\right)$ can be regarded as the matrix of the parallel transport along the family of geodesics, described above, with respect to the basis vector fields $\partial_{i}$. A vector field $Z$ which is parallel along this family of geodesics fulfils $Z=z^{A} X_{A}=\left(z^{A} \sigma_{A}^{i}\right) \partial_{i}$ with $z^{A}=$ const.

2) Important results on relations of type (1.3) have been published by $P$. Günther in [7].

3) We use the convention $R_{i j k}{ }^{\prime}=\partial_{i} \Gamma_{j k}^{l}-\partial_{j} \Gamma_{i k}^{l}+\Gamma_{i s}^{l} \Gamma_{j k}^{s}-\Gamma_{j s}^{l} \Gamma_{i k}^{s}$ with the connection coef ficients $\Gamma_{i j}^{k}=\frac{1}{2} g^{k l}\left(\partial_{i} g_{j l}+\partial_{j} g_{i l}-\partial_{l} g_{i j}\right)$. 
of all such tensor expressions which are complex linear combinations of expressions formed from the tensor coordinates in (...) by arbitrary multiplications and index contractions. Taking into account the so-called Ricci identities for the Riemannian curvature tensor

$$
\begin{aligned}
\nabla_{\{a} \nabla_{b]} \nabla_{i_{1}} \ldots \nabla_{i_{r}} R_{j_{1} \ldots j_{4}}= & -\frac{1}{2} \sum_{t=1}^{r} R_{a b i_{t}}^{m_{t}} \nabla_{i_{1}} \ldots \nabla_{m_{t}} \ldots \nabla_{i_{r}} R_{j_{1} \ldots j_{4}} \\
& -\frac{1}{2} \sum_{t=1}^{4} R_{a b j_{t}}^{m_{t}} \nabla_{i_{1}} \ldots \nabla_{i_{r}} R_{j_{1} \ldots m_{t} \ldots j_{4}}
\end{aligned}
$$

we see that the algebra $\mathcal{R}$ is generated already from $g_{i j}, g^{i j}, R_{i j k l}$ and the symmetrized covariant derivatives of the curvature tensor,

$$
\mathcal{R}=\left\langle g_{i j}, g^{i j}, R_{i j k l} ; \nabla_{\left(i_{1}\right.} \ldots \nabla_{\left.i_{r}\right)} R_{i j k l}, r \geq 1\right\rangle,
$$

because the Ricci identities yield

$$
\begin{aligned}
\nabla_{i_{1}} \ldots \nabla_{i_{r}} R_{j_{1} \ldots j_{4}}= & \nabla_{\left(i_{1}\right.} \ldots \nabla_{\left.i_{r}\right)} R_{j_{1} \ldots j_{4}} \\
& + \text { terms with covariant derivatives of } R \text { of order } r^{\prime} \leq r-2 .
\end{aligned}
$$

(We denote by (...) or [...] the symmetrization or anti-symmetrization, respectively.)

Considering (1.5) we find out that the analytic matrix function $Q$ in (1.3) depends only on the stronger symmetrized covariant derivatives

$$
\nabla_{\left(i_{1}\right.} \ldots \nabla_{i_{r}} R_{\left.|a| i_{r+1} i_{r+2}\right) b}
$$

of the curvature tensor which lie in the algebra

$$
\mathcal{R}^{s}:=\left\langle g_{i j}, g^{i j} ; \nabla_{\left(i_{1}\right.} \ldots \nabla_{i_{r}} R_{\left.|a| i_{r}+i_{r}+2\right) b}, r \geq 0\right\rangle
$$

formed from the generating tensor coordinates by the same operations like $\mathcal{R}$. (The notation $|a|$ means that the index $a$ is excluded from the symmetrization.)

Obviously, $\mathcal{R}^{s}$ is a subalgebra of $\mathcal{R}$. Now the question arises whether the algebra $\mathcal{R}^{s}$ is equal to the algebra $\mathcal{R}$. We show the equality of these two algebras by considering a more general situation.

Besides (1.3), another differential equation system allowing the calculation of the Riemannian metric from the curvature tensor in normal coordinates is given by the so-called Herglotz relations [9] which we describe in Section 2. The Herglotz relations are non-linear differential equations and yjeld power series of the metric which are determined by the symmetrized partial derivatives of the curvature tensor

$$
\partial_{\left(i_{1}\right.} \ldots \partial_{i_{r}} R_{\left.|a| i_{r+1} i_{+2}\right) b}\left(P_{0}\right)
$$

The partial derivatives of the curvature tensor $\partial_{i_{1}} \ldots \partial_{i_{r}} R_{i j k l}$ satisfy the same symmetry properties like $\nabla_{\left(i_{1}\right.} \ldots \nabla_{\left.i_{r}\right)} R_{i j k l}$ with the exception of the second Bianchi identity

$$
\nabla_{h} R_{i j k l}+\nabla_{i} R_{j h k l}+\nabla_{j} R_{h i k l}=0
$$


such that the situation given by the Herglotz relations is algebraically more general than the situation in the case of (1.3).

Using the representation theory of the symmetric group $\mathcal{S}_{r}$, we can clear up the connection between $\partial_{i_{1}} \ldots \partial_{i_{r}} R_{i j k l}$ and $\partial_{\left(i_{1}\right.} \ldots \partial_{i_{r}} R_{\left.|a| i_{r+1} i_{r}+2\right) b}$. The partial derivatives $\partial_{i_{1}} \ldots \partial_{i_{r}} R_{i j k l}$ induce group ring elements which lie in the direct sum

$$
J_{(r)} \oplus \hat{J}_{(r)} \oplus \tilde{J}_{(r)}
$$

of three minimal left ideals of $\mathbb{C}\left[\mathcal{S}_{r+4}\right]$ and the transition to the symmetrized partial derivatives $\partial_{\left(i_{1}\right.} \ldots \partial_{i,} R_{\left.|a| i_{r+1} i_{r+2}\right) b}$ corresponds to a linear mapping

$$
J_{(r)} \oplus \hat{J}_{(r)} \oplus \check{J}_{(r)} \rightarrow J_{(r)} \cdot \epsilon \quad, \quad f \mapsto f \cdot \epsilon \quad, \quad \epsilon \in \mathbb{C}\left[\mathcal{S}_{r+4}\right]
$$

which maps $\hat{J}_{(r)} \oplus \bar{J}_{(r)}$ to 0 . In the case of $\nabla_{\left(i_{1}\right.} \ldots \nabla_{\left.i_{r}\right)} R_{i j k l}$ and $\nabla_{\left(i_{1}\right.} \ldots \nabla_{i_{r}} R_{\left.|a| i_{r+1} i_{r+2}\right) b}$ only the ideals $J_{(r)}$ and $J_{(r)} \cdot \epsilon$ are associated with these covariant derivatives. The inverse mapping $J_{(r)} \cdot \epsilon \rightarrow J_{(r)}$ gives us a rclation between $\nabla_{\left(i_{1}\right.} \ldots \nabla_{\left.i_{r}\right)} R_{i j k l}$ and $\nabla_{\left(i_{1}\right.} \ldots \nabla_{i_{r}} R_{\left.|a| i_{r+1} i_{r}+2\right) b}$ which yiclds $\mathcal{R}=\mathcal{R}^{s}$.

\section{The Herglotz relations}

In this section we give a short summary of the paper [9] in which G. Herglotz states his method of determination of a Ricmannian metric from the coordinates of the Riemannian curvature tensor in normal coordinates.

Proposition 2.1. Let $(M, g)$ be an $n$-dimensional pseudo-Riemannian manifold with metric $g$ and Levi-Civita connection $\nabla$, and let $\{U, x\}$ be a system of normal coordinates on a normal neighbourhood $U \subseteq M$ with centre $P_{0} \in U$, i.e. $x\left(P_{0}\right)=0$. If we form the differential operator $X:=x^{i} \partial_{i}$ and the $(n \times n)$-matrices

$$
G:=\left(g_{i j}\right) \quad, \quad K:=\left(R_{i k l j} x^{k} x^{l}\right) \quad, \quad i \text { row index }, j \text { column index }
$$

from the coordinates $g_{i j}, R_{i k l j}$ of the metric $g$ and the Riemannian curvature tensor $R$. with respect to $\{U, x\}$, then on $U$ there holds true the so-called Herglotz relation ${ }^{1)}$

$$
X X(G)+X(G)-\frac{1}{2} X(G) \cdot G^{-1} \cdot X(G)=-2 K .
$$

Now we assume the $g_{i j}$ to be analytic functions on $U$ and make use of the facts that $\partial_{i} g_{j k}\left(P_{0}\right)=0$ in normal coordinates $\{U, x\}$ and that the metric coordinates $g_{i j}\left(P_{0}\right)$ in $P_{0}$ may be transformed into

$$
G\left(P_{0}\right)=F:=\operatorname{diag}(1, \ldots, 1,-1, \ldots,-1)
$$

by an allowed linear coordinate transformation. The numbers of 1 and -1 in the diagonal matrix $F$ are detcrmined by the signature of the metric $g$. Thus we can write $G$ as a matrix-valued power series

$$
G=F(E-\Gamma) \quad, \quad \Gamma=\sum_{k=2}^{\infty} \Gamma_{k}
$$

1) The doe". denotes the matrix product in (2.1). 
where $E$ denotes the unit matrix and the $\Gamma_{k}$ are matrix-valued homogeneous polynomials of order $k$. Equations (2.1) and (2.3) leud to

$$
X X(\Gamma)+X(\Gamma)+\frac{1}{2} X(\Gamma) \cdot(E-\Gamma)^{-1} \cdot X(\Gamma)=2 F \cdot K
$$

If we use the formulas

$$
\begin{aligned}
X\left(\Gamma_{k}\right) & =k \Gamma_{k} \\
X X\left(\Gamma_{k}\right) & =k^{2} \Gamma_{k},
\end{aligned}
$$

the Frobenius series

$$
G^{-1}=(E-\Gamma)^{-1} F=\left(E+\sum_{l=1}^{\infty} \Gamma^{l}\right) F
$$

the formula

$$
\begin{aligned}
X(\Gamma) \cdot(E-\Gamma)^{-1} \cdot X(\Gamma) & =\sum_{k, l=2}^{\infty} k l \Gamma_{k} \cdot(E-\Gamma)^{-1} \cdot \Gamma_{l} \\
& =\sum_{m=4}^{\infty} \sum_{\substack { 2 \leq k \leq\left[\frac{m}{2}\right] \\
\begin{subarray}{c}{l \\
l_{1}+\ldots+l_{k}=m \\
l_{i} \geq 2{ 2 \leq k \leq [ \frac { m } { 2 } ] \\
\begin{subarray} { c } { l \\
l _ { 1 } + \ldots + l _ { k } = m \\
l _ { i } \geq 2 } }\end{subarray}} l_{1} l_{k} \Gamma_{l_{1}} \cdot \ldots \cdot \Gamma_{l_{k}}
\end{aligned}
$$

and the power series development of $K$

$$
K=\sum_{k=2}^{\infty} K_{k}
$$

with matrix-valued homogeneous polynomials $K_{k}$ of order $k$, then we obtain the recursive relations

$$
\begin{aligned}
m=2,3: & \dot{m}(m+1) \Gamma_{m}=2 F \cdot K_{m} \\
m \geq 4: & m(m+1) \Gamma_{m}=2 F \cdot K_{m}-\frac{1}{2} \sum_{2 \leq k \leq\left[\frac{m}{2}\right]} \sum_{\substack{l_{1}+\ldots+l_{k}=m \\
l_{i} \geq 2}} l_{1} l_{k} \Gamma_{l_{1}} \ldots \cdot \Gamma_{l_{k}}
\end{aligned}
$$

In [9] G. Herglotz has proved the following facts about a metric $g$ which is determined by $(2.6)$.

Theorem 2.1. Let $\{U, x\}$ be a chart of an n-dimensional differentiable manifold $M$ with $x\left(P_{0}\right)=0$ for $P_{0} \in U$. Further let $K_{i j k l}$ be the coordinates of a covariant tensor field of order 4 which are analytic functions with respect to $\{U, x\}$ and which possess the symmetry properties of the Riemannian curvature tensor, i.e. $K_{i j k l}$ satisfies

$$
K_{i j k l}=-K_{j i k l}=-K_{i j l k}=K_{k l i j}
$$

and the first Bianchi identity

$$
K_{i j k l}+K_{i k l j}+K_{i l j k}=0 .
$$

If we consider the Herglotz rélation (2.1) with a right-hand side $K^{r}:=\left(K_{i j k l} x^{j} x^{k}\right)$ and search for a solution $G$ by means of an ansatz (2.3), then there hold true: 
1. The equations (2.6) yield a uniquely determined formal power series solution (2.3) of (2.1).

2. The convergence of this formal power series solution (2.3) follows from the convergence of the power series $K$ on a suitable open neighbourhood $U^{\prime} \subseteq U$ of $P_{0}$ by means of a comparison method.

3. The Riemannian metric $g_{i j}$ given by the calculated solution of (2.1) fulfils

$$
\left(g_{i j}-g_{i j}\left(P_{0}\right)\right) x^{j}=0,
$$

that means the coordinates $x^{i}$ are normal coordinates with respect to the constructed metric $g_{i j}$ if we restrict us to a star-shaped open neighbourhood $U^{\prime \prime} \subseteq U^{\prime}$ of $P_{0}$. The centre of these normal coordinates is $P_{0}$.

If we calculate the Riemannian curvature tensor $R_{i j k l}$ of the metric $g_{i j}$ which we have determined according to Theorem 2.1, then the Herglotz relations (2.1) hold true with $R_{i j k l}$ too such that

$$
R_{i j k l} x^{j} x^{k}=K_{i j k l} x^{j} x^{k}
$$

follows. But we will have $R_{i j k l} \neq K_{i j k l}$ in general. In the next sections we work out a characterization of the difference between $R_{i j k l}$ and $K_{i j k l}$.

\section{The decomposition of the partial derivatives of the Riemannian curvature tensor}

Although a motive of our investigations arises from techniques of differential geometry which use normal coordinates, the considerations of this paper do not require normal coordinates. If a special coordinate system is not explicitely defined, we assume always that our coordinates belong to an arbitrary chart $\{U, x\}$ of a differentiable manifold $M$.

In the following, we use statements about the connection between covariant tensors of order $r$ and the group ring $\mathbb{C}\left[\mathcal{S}_{r}\right]$ of the symmetric group $\mathcal{S}_{r}$ which we have given in [5].

Let $T$ be a covariant complex-valued tensor on a vector space $V$ on $\mathbb{C}$ and $b:=$ $\left\{v_{1}, \ldots, v_{r}\right\} \subset V$ an arbitrary subset of $r$ vectors from $V$. Then $T$ and $b$ induce a complex-valued function $T_{b}$ on the symmetric group $\mathcal{S}_{r}$

$$
T_{b}: \mathcal{S}_{r} \rightarrow \mathbb{C} \quad, \quad T_{b}: p \mapsto T_{b}(p):=T\left(v_{p(1)}, \ldots, v_{p(r)}\right)
$$

which we will identify with the group ring element $\sum_{\nu \in S_{r}} T_{b}(p) p$ denoted by $T_{b}$ too. If $T$ is a differentiable tensor field on a differentiable manifold $M$, then we obtain a group ring element $T_{b}$ for every subset $b=\left\{v_{1}, \ldots, v_{r}\right\} \subset M_{P}$ of the tangent space $M_{P}$ of any point $P \in M$.

The action of a group ring element $a=\sum_{p \in S_{r}} a(p) p \in \mathbb{C}\left[\mathcal{S}_{r}\right]$ on a tensor or a tensor field $T$ is defined by

$$
a: T \mapsto a T \quad, \quad(a T)_{i_{1} \ldots i_{r}}:=\sum_{p \in \mathcal{S}_{r}} a(p) T_{i_{p(1) \ldots i_{p(r)}}}
$$


Further, we use the mapping

$$
*: \mathbb{C}\left[\mathcal{S}_{r}\right] \rightarrow \mathbb{C}\left[\mathcal{S}_{r}\right] \quad, \quad a=\sum_{p \in \mathcal{S}_{r}} a(p) p \mapsto a^{*}:=\sum_{p \in \mathcal{S}_{r}} a(p) p^{-1}
$$

Then there holds true the relation ${ }^{1)}[5]$

$$
(a T)_{b}=T_{b} \cdot a^{*} .
$$

The power series $\left.{ }^{2}\right)$

$$
R_{i j k l}=\sum_{r=0}^{\infty} \frac{1}{r !} \partial_{i_{1}} \ldots \partial_{i_{r}} R_{i j k l}\left(P_{0}\right) x^{i_{1}} \ldots x^{i_{r}}
$$

of the Riemannian curvature tensor $\bar{R}$ around $P_{0} \in U$ is determined by the partial derivatives

$$
\left(\partial^{(r)} R\right)_{i_{1} i_{2} i_{3} i_{4} i_{5} \ldots i_{r+4}}:=\partial_{i_{5}} \ldots \partial_{i_{r+4}} R_{i_{1} i_{2} i_{3} i_{4}} \quad, \quad \partial^{(0)} R:=R
$$

of $R$ in $P \in U$. Since we will not make any coordinate transformation; we can consider the $\left(\partial^{(r)} R\right)_{i_{1} i_{2} i_{3} i_{4}, i_{5} \ldots i_{r+4}}$ as the coordinates of a 'covariant tensor field' of order $r+4$ on $U$ with respect to the basis $\left\{\partial_{1}, \ldots, \partial_{n}\right\}$ of the given chart $\{U, x\}$. Now we will investigate the left ideals of the group ring $\mathbb{C}\left[\mathcal{S}_{r+4}\right]$ in which the group ring elements $\left(\partial^{(r)} R\right)_{b}$ lie which correspond to the $\partial^{(r)} R$.

Let $r \geq 1$. We consider the stability subgroups

$$
\grave{\mathcal{S}}_{4}:=\left(\mathcal{S}_{r+4}\right)_{5, \ldots, r+4} \quad, \quad \dot{\mathcal{S}}_{r}:=\left(\mathcal{S}_{r+4}\right)_{1, \ldots, 4}
$$

of $\mathcal{S}_{r+4}$ which fix the numbers $5, \ldots, r+4$ or $1, \ldots, 4$, respectively. We denote by $\dot{y}, \dot{y}_{r}$ the group ring elements $\grave{y} \in \mathbb{C}\left[\dot{\mathcal{S}}_{4}\right], \dot{y}_{r} \in \mathbb{C}\left[\mathcal{S}_{r}\right]$ which are obtained from the Young symmetrizers of the standard tableaux ${ }^{3}$ )

$$
\begin{aligned}
& 13 \\
& 24
\end{aligned} \quad, \quad 12 \ldots(r-1) r
$$

of $\mathcal{S}_{4}, \mathcal{S}_{r}$ by means of the natural embeddings $\mathcal{S}_{4} \rightarrow \mathcal{S}_{r+4}$ and $\mathcal{S}_{r} \rightarrow \mathcal{S}_{r+4}$

$$
\begin{aligned}
& \left(\begin{array}{c}
1 \ldots 4 \\
i_{1} \ldots i_{4}
\end{array}\right) \mapsto\left(\begin{array}{c}
1 \ldots 45 \ldots r+4 \\
i_{1} \ldots i_{4} 5 \ldots r+4
\end{array}\right), \\
& \left(\begin{array}{c}
1 \ldots r \\
i_{1} \ldots i_{r}
\end{array}\right) \mapsto\left(\begin{array}{ccc}
1 \ldots 4 & 5 & \ldots r+4 \\
1 \ldots 4 i_{1}+4 \ldots i_{r}+4
\end{array}\right) \text {. }
\end{aligned}
$$

\footnotetext{
1). We use the convention $(p \circ q): i \mapsto(p \circ q)(i):=p(q(i))$ for the multiplication of permutations.

2) In (3.2) we add up on the indices $i_{1}, \ldots, i_{r}$ according to Einstein's summation convention.

3) About Young symmetrizers and Young tableaux see, for instance, $[2,5,6,10,11,13,14$, $15,16,17]$. We use the definition $y_{\mathfrak{t}}:=\sum_{p \in \mathcal{H}_{\imath}} \sum_{q \in \mathcal{V}_{\mathrm{t}}} \chi(q) p \circ q$ of a Young symmetrizer of a -:Young tableau $t$. Here- $\mathcal{H}_{t}, \mathcal{V}_{t}$ are the groups of the horizontal and vertical permutations of $t$ and $\chi(q)$ denotes the signature of the permutation $q$.
} 
Obviously, we have

$$
\begin{aligned}
\grave{y} & =\left(i d+\left(\begin{array}{ll}
1 & 3
\end{array}\right)\right) \cdot(i d+(24)) \cdot(i d-(12)) \cdot(i d-(34)) \\
\dot{y}_{r} & =\sum_{\dot{p} \in \dot{S}_{r}} \dot{p}
\end{aligned}
$$

where we have used the cyclic form of the permutations in (3.6). If $r=0$, we consider only $\dot{\mathcal{S}}_{4}=\mathcal{S}_{4}$.

Proposition 3.1. Let $\{U, x\}$ be a chart and $r \geq 1$. Then the group ring element $\left(\partial^{(r)} R\right)_{b} \in \mathbb{C}\left[\mathcal{S}_{r+4}\right]$ is contained in the left ideal

$$
I_{(r)}:=\mathbb{C}\left[\mathcal{S}_{r+4}\right] \cdot \grave{y} \cdot \dot{y}_{r}
$$

of $\mathbb{C}\left[\mathcal{S}_{r+4}\right]$ for every set of vectors $b=\left\{v_{1}, \ldots, v_{r+4}\right\} \subset M_{P}, P \in U$. If $r=0$, then every $\left(\partial^{(0)} R\right)_{b}=R_{b} \in \mathbb{C}\left[\mathcal{S}_{4}\right]$ lies in

$$
I_{(0)}:=\mathbb{C}\left[\mathcal{S}_{4}\right] \cdot \grave{y}
$$

Proof. Let $r \geq 1$. Obviously, the symmetry of $\left(\partial^{(r)} R\right)_{i_{1} i_{2} i_{3} i_{4}, i_{5} \ldots i_{r+4}}$ in $i_{5}, \ldots, i_{r+4}$ and (3.7) yield

$$
\dot{y}_{r}^{*}\left(\partial^{(r)} R\right)=\dot{y}_{r}\left(\partial^{(r)} R\right)=r ! \partial^{(r)} R .
$$

From equation (3.6) we obtain $\grave{y}^{*}\left(\partial^{(r)} R\right)$ as a sum of 16 summands for $r \geq 0$. Then we find

$$
\grave{y}^{*}\left(\partial^{(r)} R\right)=12 \partial^{(r)} R
$$

by expressing all summands of $\grave{y}^{*}\left(\partial^{(r)} R\right)$ by the two terms

$$
\left(\partial^{(r)} R\right)_{i_{1} i_{2} i_{3} i_{4}, i_{5} \ldots i_{r+4}} \quad, \quad\left(\partial^{(r)} R\right)_{i_{1} i_{3} i_{2} i_{4}, i_{5} \ldots i_{r+4}}
$$

using the identities (2.7) and (2.8). Thus there follows from (3.1), (3.10) and (3.11) for $r \geq 1$

$$
12 r !\left(\partial^{(r)} R\right)_{b}=\left(\dot{y}_{r}^{*} \grave{y}^{*}\left(\partial^{(r)} R\right)\right)_{b}=\left(\dot{\partial}^{(r)} R\right)_{b} \cdot \grave{y} \cdot \dot{y}_{r}
$$

and for $r=0$

$$
12 R_{b}=\left(\grave{y}^{*} R\right)_{b}=R_{b} \cdot \grave{y}
$$

An other proof of (3.11) follows from [6: Theorem 2.1 and remark at page 1162] (see Section 6).

Let be $r \geq 1$. We consider the representations

$$
\begin{aligned}
\grave{\alpha}: \grave{\mathcal{S}}_{4} \rightarrow G L\left(\mathbb{C}\left[\dot{\mathcal{S}}_{4}\right] \cdot \grave{y}\right) & , \quad \dot{\alpha}_{\dot{p}}(\dot{f}):=\dot{p} \cdot \dot{f} \\
\dot{\alpha}: \dot{\mathcal{S}}_{r} \rightarrow G L\left(\mathbb{C}\left[\dot{\mathcal{S}}_{r}\right] \cdot \dot{y}_{r}\right) & , \quad \dot{\alpha}_{\dot{p}}(\dot{f}):=\dot{p} \cdot \dot{f} \\
\gamma: \grave{\mathcal{S}}_{4} \cdot \dot{\mathcal{S}}_{r} \rightarrow G L\left(\left(\mathbb{C}\left[\grave{\mathcal{S}}_{4}\right] \cdot \grave{y}\right) \otimes\left(\mathbb{C}\left[\dot{\mathcal{S}}_{r}\right] \cdot \dot{y}_{r}\right)\right) & , \quad \gamma_{\dot{p} \cdot \dot{p}}(\grave{f} \cdot \dot{f}):=\dot{p} \cdot \dot{p} \cdot \dot{f} \cdot \dot{f} \\
\beta: \mathcal{S}_{r+4} \rightarrow G L\left(\mathbb{C}\left[\mathcal{S}_{r+4}\right] \cdot \grave{y} \cdot \dot{y}_{r}\right) & , \quad \beta_{p}(f):=p \cdot f
\end{aligned}
$$


Obviously, the subgroup $H:=\grave{\mathcal{S}}_{4} \cdot \dot{\mathcal{S}}_{\mathrm{r}} \subset \mathcal{S}_{\mathrm{r}+4}$ is the direct product of the subgroups $\dot{\mathcal{S}}_{4}, \dot{\mathcal{S}}_{r} \subset \mathcal{S}_{r+4}$. The tensor product in $(3.14)$ is realized by the group ring multiplication $(\dot{f}, \dot{f}) \mapsto \dot{f} \cdot \dot{f}$. This tensor product fulfils

$$
\mathbb{C}\left[\dot{\mathcal{S}}_{4} \cdot \dot{\mathcal{S}}_{r}\right] \cdot \grave{y} \cdot \dot{y}_{r}=\left(\mathbb{C}\left[\dot{\mathcal{S}}_{4}\right] \otimes \mathbb{C}\left[\dot{\mathcal{S}}_{r}\right]\right) \cdot \grave{y} \cdot \dot{y}_{r}=\left(\mathbb{C}\left[\dot{\mathcal{S}}_{4}\right] \cdot \grave{y}\right) \otimes\left(\mathbb{C}\left[\dot{\mathcal{S}}_{r}\right] \cdot \dot{y}_{r}\right) .
$$

The representation $\gamma$ is the outer tensor product of the representations $\dot{\alpha}$, $\dot{\alpha}$ (i.e. $\gamma=\dot{\alpha} \# \dot{\alpha}$ in the notation of [11]) since there holds true

$$
\gamma_{\grave{p} \cdot \dot{p}}(\grave{f} \cdot \dot{f})=(\grave{p} \cdot \dot{f}) \cdot(\dot{p} \cdot \dot{f})=\grave{\alpha}_{\grave{p}}(\grave{f}) \cdot \dot{\alpha}_{\dot{p}}(\dot{f})
$$

Further, the representations $\dot{\alpha}, \dot{\alpha}$ are irreducible because their representation spaces are left ideals generated by Young symmetrizers. Now the following lemma says that the representation $\beta$ is induced by the representation $\gamma$ (i.e. $\beta=\gamma \uparrow \mathcal{S}_{r+4}$ ).

Lemma 3.1. Let $G$ be-a finite group, $H \subseteq G$ a subgroup of $G_{-}$and_a $\in \mathbb{C}[H]$ an element of the group ring of $H$. If we consider the representations

$$
\begin{array}{cl}
\beta: G \rightarrow G L(V) & , \quad \beta_{g}(v):=g \cdot v \\
\alpha: H \rightarrow G L(W) & , \quad \alpha_{h}(w):=h \cdot w,
\end{array}
$$

with the representation spaces $V:=\mathbb{C}[G] \cdot a, W:=\mathbb{C}[H] \cdot a$, then the representation $\beta$ is induced by the representation $\alpha$, i.e. $\beta=\alpha \uparrow G$.

Proof. Obviously, there holds true $\beta_{h}(W) \subseteq W$ for all $h \in H$. We choose a system of representatives $\mathcal{R}$ of the left cosets $p \cdot H$ of $G$ relative to $H$. Let $W_{a}:=\mathcal{L}\{a\}$ be the 1 -dimensional vector space on $\mathbb{C}$ spanned by $a$. Then we can write

$$
V=\sum_{g \in G} g \cdot W_{a}=\sum_{p \in \mathcal{R}} \sum_{h \in H} p \cdot h \cdot W_{a}=\sum_{p \in \mathcal{R}} p \cdot W=\bigoplus_{p \in \mathcal{R}} \beta_{p}(W) .
$$

The last calculation step is correct because $p \cdot W \subseteq p \cdot \mathbb{C}[H]=\mathcal{L}\{p \cdot H\}$ for all $p \in \mathcal{R}$ and since $\mathbb{C}[G]=\bigoplus_{p \in \mathcal{R}} \mathcal{L}\{p \cdot H\}$

Obviously, (3.14) and (3.15) satisfy the assumptions of Lemma 3.1 since

$$
\grave{y} \cdot \dot{y}_{r} \in\left(\left(\mathbb{C}\left[\dot{\mathcal{S}}_{4}\right] \cdot \grave{y}\right) \otimes\left(\mathbb{C}\left[\dot{\mathcal{S}}_{r}\right] \cdot \dot{y}_{r}\right)\right)=\mathbb{C}\left[\grave{\mathcal{S}}_{4} \cdot \dot{\mathcal{S}}_{r}\right] \cdot \grave{y} \cdot \dot{y}_{r}
$$

Thus we obtain $\beta=\gamma \uparrow \mathcal{S}_{r+4}=(\grave{\alpha} \# \dot{\alpha}) \uparrow \mathcal{S}_{r+4}$. Now we will determine a decomposition of the left ideal $I_{(r)}$ into a direct sum of minimal left ideals (or, equivalently, a decomposition of $\beta$ into irreducible representations).

Because the representations $\dot{\alpha}, \dot{\alpha}$ are irreducible we can determine the Young frames of the irreducible subrepresentations in the decomposition of $\beta$ from the Young frames (3.5) of $\dot{\alpha}, \dot{\alpha}$ by means of the Littlewood-Richardson rule (see [13: pp. 94], [11: Vol. I, p. 84], [14: pp. 68] and [6]). From (3.5) the Littlewood-Richardson rule yields exactly the three frames

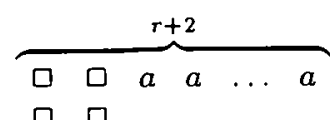

Thus we have
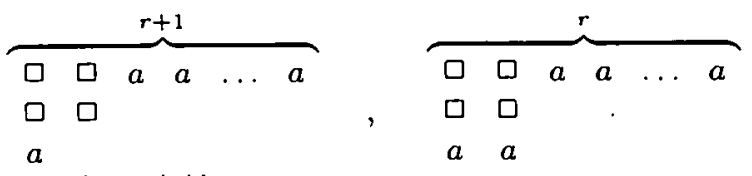
Proposition 3.2. Let $r \geq 2$. Then the representation $\beta$ according to (3.15) can be decomposed in exactly three mutually inequivalent irreducible subrepresentations which are characterized by the partitions

$$
(r+22),(r+1221),\left(\begin{array}{lll}
r & 2 & 2
\end{array}\right) \vdash r+4 .
$$

In the case $r=1$ we have only two irreducible subrepresentations given by the partitions

$$
\left(\begin{array}{ll}
3 & 2
\end{array}\right),\left(\begin{array}{lll}
2 & 2 & 1
\end{array}\right) \vdash 5 .
$$

Corollary 3.1. From Proposition 3.2 there follows:

- For $r \geq 2$ the left ideal $I_{(r)}$ can be decomposed into three mutually inequivalent minimal left ideals the equivalence classes of which are characterized by (3.16).

- For $r=1$ the left ideal $I_{(1)}$ can be decomposed into two mutually inequivalent minimal left ideals the equivalence classes of which are characterized by (3.17).

- The left ideal $I_{(0)}$ is minimal since it is generated by a Young symmetrizer.

The minimal left subideal of $I_{(r)}$ corresponding to the partition $(r+22)$ can be explicitly determined.

Proposition 3.3. Let $r \geq 0$. Then the Young symmetrizer $y_{t_{r}} \in \mathbb{C}\left[\mathcal{S}_{r+4}\right]$ of the standard tableau

$$
t_{0}:=\begin{array}{ll}
1 & 3 \\
2 & 4
\end{array} \quad, \quad t_{r}:=\begin{array}{lllllll}
1 & 3 & 5 & 6 & \ldots & (r+4) \quad, \quad r \geq 1 \\
2 & 4 & & & &
\end{array}
$$

generates that minimal left subideal $J_{(r)}$ of $I_{(r)}$ which corresponds to the partition $(r+22)$ of $r_{-}+4$.

Proof. A proof is necessary only for $r \geq 1$. We show that there is a $c=$ const $\neq 0$ such that

$$
y_{t_{r}} \cdot \dot{y} \cdot \dot{y}_{r}=c y_{t_{r}}
$$

Then there follows from (3.19) that the minimal left ideal $K_{(r)}:=\mathbb{C}\left[\mathcal{S}_{r+4}\right] \cdot y_{t_{r}}$ is a subideal of $I_{(r)}$. But because the decomposition of $I_{(r)}$ into a direct sum of minimal left ideals contains exactly one minimal left ideal $J_{(r)}$ corresponding to the partition $(r+22)$, the ideal $K_{(r)}$ has to coincide with that ideal $J_{(r)}$.

Let us prove (3.19). We denote by $P_{\left\{i_{1}, \ldots, i_{k}\right\}}$ the subgroup of $\mathcal{S}_{r+4}$ consisting of all those permutations from $\mathcal{S}_{r+4}$ which fix all numbers in $\{1, \ldots, r+4\} \backslash\left\{i_{1}, \ldots, i_{k}\right\}$. Now let $\mathcal{H}_{t_{r}}$ be the group of the horizontal permutations of the tableaux $t_{r}$ and let $\mathcal{R}$ be a system of representatives of the left cosets of $P_{\{1,3,5,6, \ldots, r+4\}}$ relative to $P_{\{1,3\}}$. Then we can write

$$
\sum_{p \in \mathcal{H}_{i_{r}}} p=\sum_{s \in \mathcal{R}} s \cdot(i d+(13)) \cdot(i d+(24))
$$


and

$$
\begin{aligned}
& y_{t_{r}}=\sum_{s \in \mathcal{R}} s \cdot(i d+(13)) \cdot(i d+(2 s)) \cdot(i d-(12)) \cdot(i d-(34)), \\
& y_{t_{r}}=\sum_{s \in \mathcal{R}} s \cdot \grave{y} .
\end{aligned}
$$

Since $\grave{y} \cdot \grave{y}=\mu \grave{y}$ with a constant $\mu \neq 0$, we obtain from (3.20)

$$
y_{t_{r}} \cdot \dot{y} \cdot \dot{y}_{r}=\sum_{s \in \mathcal{R}} s \cdot \grave{y} \cdot \dot{y} \cdot \dot{y}_{r}=\mu \sum_{s \in \mathcal{R}} s \cdot \grave{y} \cdot \dot{y}_{r}=\mu y_{t_{r}} \cdot \dot{y}_{r} .
$$

Now let $\tilde{\mathcal{R}}$ be a system of representatives of the left cosets of $P_{\{1,3,5,6, \ldots, r+4\}}$ relative to $P_{\{5,6, \ldots, r+4\} \text {. Then there holds }}$

$$
\sum_{p \in \mathcal{H}_{t_{r}}} p=\sum_{\tilde{s} \in \overline{\mathcal{R}}} \tilde{s} \cdot \dot{y}_{r} \cdot(i d+(24))=(i d+(24)) \cdot \sum_{\bar{s} \in \tilde{\mathcal{R}}} \tilde{s} \cdot \dot{y}_{r} .
$$

Denoting the group of vertical permutations of $t_{r}$ by $\mathcal{V}_{t_{r}}$ and taking into account that $\dot{y}_{r} \cdot q=q \cdot \dot{y}_{r}$ for all $q \in \mathcal{V}_{t_{r}}$, we can write

$$
y_{t_{r}}=\sum_{p \in \mathcal{H}_{t_{r}}} \sum_{q \in \mathcal{V}_{t_{r}}} \chi(q) p \cdot q=(i d+(24)) \cdot \sum_{\tilde{s} \in \overline{\mathcal{R}}} \sum_{q \in \mathcal{V}_{z_{r}}} \chi(q) \tilde{s} \cdot q \cdot \dot{y}_{r} .
$$

Then this relation and $(3.21)$ yield

$$
\begin{aligned}
y_{t_{r}} \cdot \dot{y} \cdot \dot{y}_{r} & =\mu(i d+(24)) \cdot \sum_{\dot{s} \in \overline{\mathcal{R}}} \sum_{q \in \mathcal{V}_{t_{r}}} \chi(q) \tilde{s} \cdot q \cdot \dot{y}_{r} \cdot \dot{y}_{r} \\
& =\mu r !(i d+(24)) \cdot \sum_{\bar{s} \in \mathcal{R}} \sum_{q \in \mathcal{V}_{t_{r}}} \chi(q) \tilde{s} \cdot q \cdot \dot{y}_{r} \\
& =\mu r ! y_{t_{r}}
\end{aligned}
$$

\section{The essential part of the partial derivatives of the Riemannian curvature tensor}

Since the right-hand side of the Herglotz relation is the matrix with elements $R_{i j k l} x^{j} x^{k}$, the Riemannian metric $g$ does not depend on the partial derivatives $\partial_{i_{1}} \ldots \partial_{i_{r}} R_{i j k l}\left(P_{0}\right)$ of the Riemannian curvature tensor but on the symmetrized partial derivatives

$$
\begin{gathered}
\left(\partial^{(r)} \breve{R}\right)_{i_{1} i_{2} i_{3} i_{4} i_{5} \ldots i_{r+4}}:=\partial_{\left(i_{5} \ldots \partial_{i_{r+4}} R_{\left.i_{i_{1}} i_{2} i_{3}\right) i_{4}}\right.} \\
\left(\partial^{(0)} \breve{R}\right)_{i_{1} \ldots i_{4}}:=\breve{R}_{i_{1} \ldots i_{4}}:=R_{i_{1}\left(i_{2} i_{3}\right) i_{4}}
\end{gathered}
$$

of the curvature tensor at the centre $P_{0}$ of the normal neighbourhood $U$.

Let now $\{U, x\}$ be an arbitrary chart which do not have to be a normal coordinate system. In this section we investigate the left ideal of $\mathbb{C}\left[\mathcal{S}_{r+4}\right]$ which contains the group ring elements $\left(\partial^{(r)} \breve{R}\right)_{b}$ induced by $\partial^{(r)} \breve{R}$ and a vector set $b^{\circ}=\left\{v_{1}, \ldots v_{r+4}\right\} \subset M_{P}$, $P \in U$. 
Lemma 4.1. Let be $r \geq 0$. We denote by $C$ the subgroup of $\mathcal{S}_{r+4}$ which fixes the numbers 1 and 4 and by $\epsilon$ the sum of all elements of $C$,

$$
C:=P_{\{2,3,5, \ldots, r+4\}} \quad, \quad \epsilon:=\sum_{c \in C} c .
$$

Then the group ring element $\left(\partial^{(r)} \breve{R}\right)_{b}$ induced by $\partial^{(r)} \breve{R}$ and $a$ set $b=\left\{v_{1}, \ldots, v_{r+4}\right\} \subset$ $M_{P}$ of vectors of the tangent space $M_{P}$ lies in the left ideal $\breve{I}_{(r)}:=I_{(r)} \cdot \epsilon$ of $\mathbb{C}\left[S_{r+4}\right]$ for every vector set $b$.

Proof. Because there holds true $\partial^{(r)} \breve{R}=\epsilon\left(\partial^{(r)} R\right) /(r+2)$ ! and $\epsilon^{*}=\epsilon$ we obtain the assertion from

$$
\left(\partial^{(r)} \breve{R}\right)_{b}=\frac{1}{(r+2) !}\left(\epsilon\left(\partial^{(r)} R\right)\right)_{b}=\frac{1}{(r+2) !}\left(\partial^{(r)} R\right)_{b} \cdot \epsilon
$$

We consider the decomposition of $I_{(r)}$ into minimal left ideals

$$
I_{(\mathbf{r})}=J_{(r)} \oplus \hat{J}_{(r)} \oplus \tilde{J}_{(r)}
$$

according to Corollary 3.1. Let the correspondence between the minimal left ideals and their characterizing partitions be

$$
\begin{aligned}
& J_{(r)} \Leftrightarrow\left(\begin{array}{lll}
r+2 & 2
\end{array}\right), \\
& \hat{J}_{(r)} \Leftrightarrow\left(\begin{array}{lll}
r+1 & 2 & 1
\end{array}\right), \\
& \tilde{J}_{(r)} \Leftrightarrow\left(\begin{array}{lll}
r & 2 & 2
\end{array}\right) .
\end{aligned}
$$

If $r=1$, then $\check{J}_{(r)}$ does not occur in (4.4).

From (4.4) there follows a decomposition of $\breve{I}_{(r)}$

$$
\breve{I}_{(r)}=\left(J_{(r)} \cdot \epsilon\right) \oplus\left(\hat{J}_{(r)} \cdot \epsilon\right) \oplus\left(\check{J}_{(r)} \cdot \epsilon\right)
$$

which is certainly a direct sum since the minimal left ideals are mutually inequivalent. Now the question arises whether one of the ideals $\left(J_{(r)} \cdot \epsilon\right),\left(\hat{J}_{(r)} \cdot \epsilon\right),\left(\breve{J}_{(r)} \cdot \epsilon\right)$ vanishes.

Theorem 4.1. For $r \geq 0$ there holds true

$$
\breve{I}_{(r)}=J_{(r)} \cdot \epsilon=\mathbb{C}\left[\mathcal{S}_{r+4}\right] \cdot y_{t_{r}} \cdot \epsilon
$$

that means all other minimal left ideals in (4.4) are mapped to 0 by $f \mapsto f \cdot \epsilon$.

Proof. Step 1 : First we show that $y_{t_{r}} \cdot \epsilon \neq 0$. We use the notations $t_{r}, \mathcal{H}_{t_{r}}, \mathcal{V}_{t_{r}}$ of Section 3. Denoting $C^{\prime}:=P_{\{1,3\}}$ if $r=0, C^{\prime}:=P_{\{1,3,5, \ldots, r+4\}}$ if $r \geq 1$ and taking into account $C^{\prime}=(12) \cdot C \cdot(12)$ we can write for the sum of the horizontal permutations of the tableaux $t_{r}(3.18)$

$$
\sum_{p \in \mathcal{H}_{\mathrm{t}_{r}}} p=\sum_{s \in C^{\prime}} s+\sum_{s \in C^{\prime}} s \cdot(24)=(12) \cdot \epsilon \cdot(12) \cdot(i d+(24))
$$


Because (12) is a vertical permutation of $t_{r}$, there follows on the other hand

$$
y_{t_{r}} \cdot(12)=\sum_{q \in \mathcal{V}_{t_{r}}} \sum_{p \in \mathcal{H}_{t_{r}}} \chi(q) p \cdot q \cdot(12)=\chi((12)) \sum_{q \in \mathcal{V}_{t_{r}}} \sum_{p \in \mathcal{H}_{t_{r}}} \chi(q) p \cdot q=-y_{t_{r}} .
$$

Thus we obtain

$$
\begin{aligned}
y_{t_{r}} \cdot y_{t_{r}} & =\sum_{q \in \mathcal{V}_{t_{r}}} \sum_{p \in \mathcal{H}_{t_{r}}} \chi(q) y_{t_{r}} \cdot p \cdot q \\
& =y_{t_{r}} \cdot(12) \cdot \epsilon \cdot(12) \cdot(i d+(24)) \cdot \sum_{q \in \mathcal{V}_{t_{r}}} \chi(q) q \\
& =-y_{t_{r}} \cdot \epsilon \cdot(12) \cdot(i d+(24)) \cdot \sum_{q \in \mathcal{V}_{t_{r}}} \chi(q) q .
\end{aligned}
$$

But this yields $y_{t_{r}} \cdot \epsilon \neq 0$ since $y_{t_{r}} \cdot y_{t_{r}} \neq 0$. Consequently, the ideal $J_{(r)} \cdot \epsilon$ has to occur in the decomposition (4.5).

If $r=0$, Theorem 4.1 follows from $I_{(0)}={ }^{\prime} J_{(0)}$. Thus we can assume $r \geq 1$ in the following.

Step 2: Using the hook length formula (see [11: Vol I, p. 81], [1: pp. 101] and [6]) we can calculate the dimensions of the left ideals $J_{(r)}, \hat{J}_{(r)}, \bar{J}_{(r)}$ from the Young frames of these ideals or, equivalently, from the partitions (3.16). The results are

$$
\begin{aligned}
& r \geq 0 \Rightarrow d_{r}:=\operatorname{dim} J_{(r)}=\frac{(r+4)(r+1)}{2}, \\
& r \geq 1 \Rightarrow \hat{d}_{r}:=\operatorname{dim} \hat{J}_{(r)}=\frac{(r+4)(r+2) r}{3}, \\
& r \geq 2 \Rightarrow \quad \dot{d}_{r}:=\operatorname{dim} \bar{J}_{(r)}=\frac{(r+4)(r+3) r(r-1)}{12} \text {. }
\end{aligned}
$$

Furthermore, the left ideal $L_{(r)}:=\mathbb{C}\left[\mathcal{S}_{r+4}\right] \cdot \epsilon$ has the dimension

$$
l_{r}:=\operatorname{dim} L_{(r)}=(r+4)(r+3) \text {. }
$$

Consider a system of representatives $\mathcal{R}$ of the left cosets of $\mathcal{S}_{r+4}$ relative to $C$. Then $\mathcal{B}:=\{p \cdot \epsilon \mid p \in \mathcal{R}\}$ is a system of generating vectors of $L_{(r)}$. But on the other hand $\mathcal{B}$ is a system of linearly independent vectors since the vectors $p \cdot \epsilon$ lie in pairwise distinct cosets. Thus $\mathrm{B}$ has a basis of $|\mathcal{R}|=(r+4)(r+3)$ vectors.

The left ideal $\breve{I}_{(r)}$ is a subideal of $L_{(r)}$ such that $\operatorname{dim} \breve{I}_{(r)} \leq \operatorname{dim} L_{(r)}$. Further, the linear mapping $f \mapsto f \cdot \epsilon$ maps a minimal left ideal either onto 0 or onto an equivalent minimal left ideal. In Table 1 we have listed the first values of the dimensions $d_{r}, \hat{d}_{r}, \bar{d}_{r}, l_{r}$. Since these dimensions are monotonically increasing functions of $r$ and $\breve{I}_{(r)}$ has a subideal of dimension $d_{r}$ for all $r \geq 1$, we read from Table 1 that for $r \geq 4$ subideals of dimensions $\hat{d}_{r}, \dot{d}_{r}$ can not occur in $\breve{I}_{(r)}$. Moreover, for $r=3$ a subideal of $\breve{I}_{(r)}$ of dimension $\hat{d}_{3}=35$ is impossible.

Step 3: We handle the remaining cases of the left ideals $\hat{J}_{(1)}^{-}, \hat{J}_{(2)}, \dot{J}_{(2)}, \dot{J}_{(3)}$ by a 
Table 1. The dimensions $d_{r}, \hat{d}_{r}, \dot{d}_{r}, l_{r}$ for low $r$.

\begin{tabular}{rrrrr}
\hline$r$ & $d_{r}$ & $\dot{d}_{r}$ & $\dot{d}_{r}$ & $l_{r}$ \\
\hline 1 & 5 & 5 & $/$ & 20 \\
2 & 9 & 16 & 5 & 30 \\
3 & 14 & 35 & 21 & 42 \\
4 & 20 & 64 & 56 & 56 \\
5 & 27 & 105 & 120 & 72 \\
\hline
\end{tabular}

computer calculation applying our Mathematica package PERMS [4]. To determine generating idempotents of these left ideals we consider the Young standard tableaux

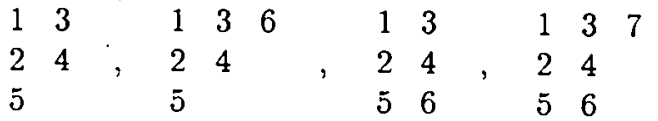

Let $y$ run through the set of the four Young symmetrizers of the tableaux (4.11). Then we find by means of PERMS

$$
y \cdot \grave{y} \cdot \dot{y}_{r} \neq 0 \text { and } y \cdot \dot{y} \cdot \dot{y}_{r} \cdot y \neq 0
$$

for all those four Young symmetrizers $y$. There follows from the second of these relations that $y \cdot \grave{y} \cdot \dot{y}_{r}$ is an essentially idempotent element generating a minimal left subideal of $I_{(r)}$ of the equivalence class of $y^{1)}$. But since $I_{(r)}$ has at most one subideal from the equivalence class of $y$, these essentially idcmpotent elements are generating clements of the left ideals $\hat{J}_{(1)}, \hat{J}_{(2)}, \breve{J}_{(2)}, \breve{J}_{(3)}$. Now another calculation with PERMS yields

$$
y \cdot \grave{y} \cdot \dot{y}_{r} \cdot \epsilon=0
$$

for all $y$. Thus the ideals $\left(\hat{J}_{(1)} \cdot \epsilon\right),\left(\hat{J}_{(2)} \cdot \epsilon\right),\left(\breve{J}_{(2)} \cdot \epsilon\right),\left(\vec{J}_{(3)} \cdot \epsilon\right)$ vanish

Definition 4.1. Let,$y_{t_{r}}$ be the Young symmetrizer of the standard tableau (3.18). We call $y_{t_{r}}^{*}\left(\partial^{(r)} R\right)$ the essential part of $\partial^{(r)} R$ and $\partial^{(r)} R-y_{t_{r}}^{*}\left(\partial^{(r)} R\right)$ the non-essential part of $\partial^{(r)} R$.

Obviously, the mapping $f \mapsto f \cdot \epsilon$ is an isomorphism of the minimal left ideals $J_{(r)}$ and $\left(J_{(r)} \cdot \epsilon\right)$, describing the equivalence of these ideals. From this fact there follows

$$
\partial^{(r)} \breve{R}=\text { const } \epsilon\left(y_{i_{r}}^{*}\left(\partial^{(r)} R\right)\right) ;, \text { const } \neq 0 .
$$

We finish this section with a formula for the inverse of this mapping.

Proposition 4.1. Let $r \geq 0$ and denote $y_{t_{r}}$ the Young symmetrizer of the Young tableau (3.18) and $\epsilon$ the group ring element according to (4.3). Let further be $e^{2)}$

$$
\eta .:=(12) \cdot(i d+(24)) \cdot(i d-(12)) \cdot(i d-(34))
$$

\footnotetext{
1) This situation is a special case of Proposition 3.1 in [5].

2) In the cyclic form of a permutation we write the image of a number left from the inverse image.
} 
Then there holds true

$$
y_{t_{r}} \cdot \epsilon \cdot \eta=-\mu_{r} y_{t_{r}} \cdot \text { with } \quad \mu_{r}:=2(r+3)(r+2) r !
$$

such that the mapping $J_{(r)} \cdot \epsilon \rightarrow J_{(r)}, h \mapsto-\left(1 / \mu_{r}\right) h \cdot \eta$ is the inverse of the mapping $J_{(r)} \rightarrow J_{(r)} \cdot \epsilon, f \mapsto f \cdot \epsilon:$ From (4.13), (4.14) there follows

$$
\begin{aligned}
& \frac{1}{(r+2) !}\left(y_{i_{r}}^{*}\left(\partial^{(r)} R\right)\right)_{i_{1} i_{2} i_{3} i_{4} i_{3} \ldots i_{r+4}}= \\
& +\left(\partial^{(r)} \breve{R}\right)_{i_{1} i_{2} i_{3} i_{4} i_{5} \ldots i_{r+4}}-\left(\partial^{(r)} \breve{R}\right)_{i_{2} i_{1} i_{3} i_{4} i_{5} \ldots i_{r+4}}-\left(\partial^{(r)} \breve{R}\right)_{i_{1} i_{2} i_{4} i_{3} i_{5} \ldots i_{r+4}} \\
& +\left(\partial^{(r)} \breve{R}\right)_{i_{4} i_{2} i_{3} i_{1} i_{5} \ldots i_{r+4}}+\left(\partial^{(r)} \breve{R}\right)_{i_{2} i_{1} i_{4} i_{3} i_{5} \ldots i_{r+4}}-\left(\partial^{(r)} \breve{R}\right)_{i_{4} i_{1} i_{3} i_{2} i_{5} \ldots i_{r+4}} \\
& -\left(\partial^{(r)} \breve{R}\right)_{i_{3} i_{2} i_{4} i_{1} i_{5} \ldots i_{r+4}}+\left(\partial^{(r)} \breve{R}\right)_{i_{3} i_{1} i_{4} i_{2} i_{5} \ldots i_{r+4}} .
\end{aligned}
$$

Proof. Equation (4.14) follows from (4.6), definition (4.13), equation (4.7) and

$$
y_{t_{r}} \cdot y_{t_{r}}=\mu_{r} y_{t_{r}} \quad \text { with } \quad \mu_{r}:=\frac{(r+4) !}{d_{r}}, d_{r}:=\operatorname{dim} J_{(r)}
$$

The formula for $\mu_{r}$ in (4.16) is given, e.g., in [1: p. 103].

We denote by $e, \hat{e}, \bar{e}$ the generating idempotents of $J_{(r)}, \hat{J}_{(r)}, \check{J}_{(r)}$ corresponding to the decomposition (4.4) of $I_{(r)}$. These idempotents fulfil

$$
e=\frac{1}{\mu_{r}} y_{t_{r}} \quad, \quad \hat{e} \cdot \epsilon=0, \quad \check{e} \cdot \epsilon=0 .
$$

Furthermore, we can write for every vector set $b=\left\{v_{1}, \ldots, v_{r+4}\right\} \subset M_{P}$ of the tangent space $M_{l}$,

$$
\left(\partial^{(r)} R\right)_{b}=\left(\partial^{(r)} R\right)_{b} \cdot e+\left(\partial^{(r)} R\right)_{b} \cdot \dot{e}+\left(\partial^{(r)} R\right)_{b}^{(r)} \cdot \bar{e}
$$

Then using equation (4.14), (4.17) and $\epsilon^{*}\left(\partial^{(r)} R\right)=(r+2) ! \partial^{(r)} \breve{R}$ we obtain

$$
\begin{aligned}
\left(y_{\iota_{+}}^{*}\left(\partial^{(r)} R\right)\right)_{b} & =\left(\partial^{(r)} R\right)_{b} \cdot y_{t_{r}}=-\left(\partial^{(r)} R\right)_{b} \cdot e \cdot \epsilon \cdot \eta=-\left(\partial^{(r)} R\right)_{b} \cdot \epsilon \cdot \eta \\
& =-\left(\epsilon^{*}\left(\partial^{(r)} R\right)\right)_{b} \cdot \eta=-(r+2) !\left(\partial^{(r)} \breve{R}\right)_{b} \cdot \eta \\
& =-(r+2) !\left(\eta^{*}\left(\partial^{(r)} \breve{R}\right)\right)_{b}
\end{aligned}
$$

and consequently

$$
y_{i}^{*}\left(\partial^{(\cdot)} R\right)=-(r+2) ! \eta^{*}\left(\partial^{(r)} \breve{R}\right)
$$

This together with

$$
\eta^{*}=-i d+(12)+(34)-(14)-(12)(34)+(124)+(143)-(1243)
$$

yields $(4.1 \bar{j})$ 


\section{The occurrence of the non-essential part of the partial derivatives of the Riemannian curvature tensor}

In this section we discuss the question whether examples of metrics can be found for which the $\left(\partial^{(r)} R\right)_{b}$ of the partial derivatives of the curvature tensor possesses nonvanishing parts lying at least in one of the left ideals $\hat{J}_{(r)}$ or $\dot{J}_{(r)}$. First we give a case for which the $\left(\partial^{(r)} R\right)_{b}$ are contained exclusively in $J_{(r)}$.

Proposition 5.1. We assume that the Riemannian metric $g$ is decomposable into a sum of 2-dimensional metrics $g^{(i)}, i=1, \ldots, m$, that means around every point $P_{0}$ of the underlying manifold $M$ a chart $\{U, x\}$ can be found such that the metric takes the form

$$
\begin{array}{cl}
d s^{2}=g_{\alpha \beta} d x^{\alpha} d x^{\beta}= & \sum_{i=1}^{m} g_{\alpha_{i} \beta_{i}}^{(i)}\left(x^{\gamma_{i}}\right) d x^{\alpha_{i}} d x^{\beta_{i}} \\
\alpha, \beta \in\{1, \ldots, 2 m\} & \alpha_{i}, \beta_{i}, \gamma_{i} \in\{2 i-1,2 i\}
\end{array}
$$

Then there holds true with respect to $\{U, x\}$

$$
\left(\partial^{(r)} R\right)_{b} \in J_{(r)}=\mathbb{C}\left[\mathcal{S}_{r}\right] \cdot y_{\iota_{r}}
$$

for $r \geq 1$ and every $b=\left\{v_{1}, \ldots, v_{r+4}\right\} \subset M_{P}, P \in U$. In particular, a 2-dimensional Riemannian manifold fulfils (5.2).

Proof. If we calculate the Christoffel symbols and the coordinates of the curvature tensor and its partial derivatives for a decomposable metric (5.1), we obtain that at most those coordinates

$$
\Gamma_{\mu_{i} \nu_{i}}^{\kappa_{i}}\left(x^{\gamma_{i}}\right), R_{\kappa_{i} \lambda_{i} \mu_{i} \nu_{i}}\left(x^{\gamma_{i}}\right), \partial_{\alpha_{1}} R_{\kappa_{i} \lambda_{i} \mu_{i} \nu_{i}}\left(x^{\gamma_{i}}\right), \partial_{\alpha_{i}} \partial_{\beta_{i}} R_{\kappa_{1} \lambda_{i} \mu_{i} \nu_{i}}\left(x^{\gamma_{i}}\right), \ldots
$$

do not vanish, the indices of which lie in one of the sets $\{2 i-1,2 i\}$, i.e.

$$
\alpha_{i}, \beta_{i}, \dot{\gamma}_{i}, \kappa_{2}, \dot{\lambda}_{i}, \mu_{i}, \nu_{2} \in\{2 i-1,2 i\} \quad, \quad i=1, \ldots, m
$$

As in the proof of Proposition 4.1 we denote by $e, \hat{e}, \bar{e}$ the generating idempotents of $J_{(r)}, \hat{J}_{(r)}, \tilde{J}_{(r)}$ corresponding to the decomposition (4.4) of $I_{(r)}$. The left ideal $\hat{J}_{(r)}$ belongs to the equivalence class of minimal left ideals of the partition $\lambda=(r+121)$. The left ideal

$$
I_{\lambda}:=\bigoplus_{t \in S \tau_{\lambda}} \mathbb{C}\left[\mathcal{S}_{r}\right] \cdot y_{t}
$$

contains all minimal left ideals of the class of $\lambda$ (see, e.g., [1: p.58 and p.102]). In (5.3) $\mathcal{S} \mathcal{T}_{\lambda}$ denotes the set of all standard tableaux of the partition $\lambda$ and $y_{t}$ is the Young symmetrizer of the standard tableau $t$. Since $\hat{e} \in I_{\lambda}$, we can writc

$$
\hat{e}=\sum_{t \in \mathcal{S} \tau_{\lambda}} x_{\ell} \cdot y_{t}
$$


with certain group ring elements $x_{\imath} \in \mathbb{C}\left[\mathcal{S}_{r}\right]$.

Now, equation (5.4) yields

$$
\hat{e}^{*}\left(\partial^{(r)} R\right)=\sum_{t \in S T_{\lambda}} y_{t}^{*}\left(x_{t}^{*}\left(\partial^{(r)} R\right)\right)
$$

$x_{t}^{*}\left(\partial^{(r)} R\right)$ is a linear combination of certain coordinates of $\partial^{(r)} R$ with permuted indices. The application of $y_{i}^{*}$ to $x_{i}^{*}\left(\partial^{(r)} R\right)$ brings an anti-symmetrization of three indices about every summand of $x_{i}^{*}\left(\partial^{(r)} R\right)$ because every standard tableaux $t \in S \mathcal{S} \mathcal{T}_{\lambda}$ has three rows. But a non-vanishing coordinate of $\partial^{(r)} R$ can not have more than two values among its indices, so $y_{t}^{*}\left(x_{t}^{*}\left(\partial^{(r)} R\right)\right)=0$ for all $t \in S \mathcal{T}_{\lambda}$. Consequently, there follows $\hat{e}^{*}\left(\partial^{(r)} R\right)=0$ and $\left(\partial^{(r)} R\right)_{b} \cdot \hat{e}=0$ for all vector sets $b=\left\{v_{1}, \ldots, v_{r+4}\right\} \subset M_{P}$.

By the same arguments we can show that $\left(\partial^{(r)} R\right)_{b} \cdot \bar{e}=0$ for all $b=\left\{v_{1}, \ldots, v_{r+4}\right\} \subset$ $M_{P}$. Taking into account (4.17), we obtain $\left(\partial^{(r)} R\right)_{b}=\left(\partial^{(r)} R\right)_{b} \cdot c \in J_{(r)}$

An cxample of a metric such that $\left(\partial^{(r)} R\right)_{b}$ have a part in the ideal $\hat{J}_{(r)} \oplus \tilde{J}_{(r)}$ can be found in the class of Riemannian manifolds for which the $R_{i j k l} x^{j} x^{k}$ are polynomials in normal coordinates $x^{i}$.

Proposition 5.2. Let $\{U, x\}$ be a chart of a 3-dimensional analytic manifold with $x\left(P_{0}\right)=0$ for a point $P_{0} \in U$. Consider the Herglotz relations (2.1) with a right-hand side

$$
K=\left(K_{i j k l} x^{j} x^{k}\right) \text { with } K_{i j k l}:=\delta_{i l} \delta_{j k}-\delta_{i k} \delta_{j l}, \quad \delta_{i j}:=\left\{\begin{array}{lll}
1 & \text { if } & i=j \\
0 & \text { if } & i \neq j
\end{array}\right.
$$

If we determine the formal power series solution $G$ of (2.1) to a positive definite metric $g$ from (2.6) and choose $e^{1)}$ an open noighbourhood $U^{\prime} \subseteq U$ of $P_{0} \in U$ such that the series of $G$ converges on $U^{\prime}$ and the chart $\left\{U^{\prime}, x\right\}$ is a normal coordinate system of the metric $g$, then the Riemannian curvature tensor $R$ of the calculated metric $g$ fulfils

$$
\forall r \geq 1, \forall b=\left\{v_{1}, \ldots, v_{r+4}\right\} \subset M_{P_{0}}: \quad\left(\partial^{(r)} R\right)_{b} \in \tilde{J}_{(r)} \oplus \check{J}_{(r)}
$$

Furthermore, there holds $\left(\partial^{(v)} R\right)_{6} \neq 0$ at least for $r=2,4,6$ and for suitable chosen vector sets $b=\left\{v_{1}, \ldots, v_{r+1}\right\} \subset M_{P_{0}}$.

Proof. Obviously, the matrix $K$ from (5.5) satisfies

$$
K \cdot K=r^{2} K \text { with } r:=\sqrt{\left(x^{1}\right)^{2}+\left(x^{2}\right)^{2}+\left(x^{3}\right)^{2}} .
$$

Taking into account $(5.7)$ and $F=E=\left(\delta_{i j}\right), K_{2}=K, K_{m}=0$ for $m \geq 3$ we obtain from (2.0)

$$
\left.\begin{array}{l}
\Gamma_{2 m+1}=0 \\
\Gamma_{2 m}=c_{m}, r^{2 m-2} K \quad, \quad c_{m}=\text { const }
\end{array}\right\} \quad, \quad m=1,2, \ldots
$$

1) This is possible on the hasis of 'Theorem 2.1 . 
This yields

$$
G=E+f(r) K
$$

with a convergent power series $f(r)$ for wich a more precise calculation ${ }^{1)}$ gives

$$
f(r)=-\frac{1}{3}+\frac{1}{90} r^{2}+\frac{1}{945} r^{4}+\frac{43}{340200} r^{6}+\ldots
$$

The metric $g_{i j}$ defined by (5.9) is centrally symmetric and turns into

$$
d s^{2}=d r^{2}+h(r)\left\{d \theta^{2}+\sin ^{2} \theta d \dot{\phi}^{2}\right\} \quad, \quad h(r):=r^{2}+r^{4} f(r)
$$

if we introduce spherical coordinates

$$
x^{1}=r \cos \phi \sin \theta, x^{2}=r \sin \phi \sin \theta \quad ; \quad x^{3}=r \cos \theta .
$$

The non-vanishing Christoffel symbols of a metric (5.11) are

$$
\begin{aligned}
& \Gamma_{\theta \theta}^{r}=-\frac{1}{2} h^{\prime}(r) \quad ; \Gamma_{r \theta}^{\theta}=\frac{h^{\prime}(r)}{2 h(r)} \quad ; \Gamma_{r \dot{\phi}}^{\phi}=\frac{h^{\prime}(r)}{2 h(r)} \\
& \Gamma_{\phi 0}^{r}=-\frac{1}{2} h^{\prime}(r) \sin ^{2} \theta, \Gamma_{\dot{\phi} \phi}^{\theta}=-\sin \theta \cos \theta, \Gamma_{\theta \phi}^{\dot{\phi}}=\cot \theta .
\end{aligned}
$$

The only non-vanishing coordinates of the curvature tensor of (5.11) read ${ }^{2)}$

$$
R_{r \theta r \theta}=\frac{h^{\prime \prime}(r)}{2}-\frac{h^{\prime}(r)^{2}}{4 h(r)}, R_{r \phi r \phi}=R_{r \theta r \theta} \sin ^{2} \theta: R_{\theta \dot{\theta} \phi}=\left(\frac{h^{\prime}(r)^{2}}{4}-h(r)\right) \sin ^{2} \theta
$$

Now we calculate from (5.10) and (5.11)

$$
\frac{h^{\prime}(r)^{2}}{4}-h(r)=-r^{4}+\frac{1}{2} r^{6}-\frac{1}{27} r^{8}-\frac{11}{3240} r^{10}+\ldots
$$

Since the coordinate transformation

$$
R_{\theta \phi \theta \dot{0}}=\partial_{\theta} x^{i} \partial_{\phi} x^{j} \partial_{\theta} x^{k} \partial_{\phi} x^{l} R_{i j k l} \quad ; \quad i, j, k, l \in\{1,2,3\}
$$

produces a multiplication of the coordinates $R_{i j k l}$ relating to $\left\{U^{\prime}, x\right\}$ by a factor $r^{4}$, we see from (j.12) that the power series of the coordinates $R_{i j k l}$ contain homogeneous polynomials of orders 2,4 and 6 in the coordinates $x^{1}, x^{2}, x^{3}$. From this there follows $\left.\partial^{(m)} R\right|_{P_{0}} \neq 0$ for $m=2,4,6$.

But because $R_{i j k l} x^{j} x^{k}$ is a quadratic polynomial in the coordinates $x^{i}$ we have $\left.\partial^{(m)} \check{R}\right|_{P_{0}}=0$ for $m \geq 1$. Then (4.1j) yields $\left.y_{i_{m}}^{*}\left(\partial^{(m)} R\right)\right|_{P_{0}}=0$ and consequently $\left(\partial^{(m)} R\right)_{b} \in j_{(m)} \subseteq j_{(m)}$ for all $b=\left\{v_{1}, \ldots, v_{m+4}\right\} \subset M_{P_{0}}$ and $m \geq 1$. Furthermore, there exist non-vanishing group ring elements $\left(\partial^{(m)} R\right)_{b}$ for at least $m=2,4,6$ since $\left.\partial^{(m)} R\right|_{P_{0}} \neq 0$ for these $m$-values

\footnotetext{
1) We have done the calculations of (5.9) and (5.12) by means of Mathematica [18].

2) The $\Gamma_{\mu \nu}^{\kappa}$ and the $R_{\lambda \kappa \mu \nu}$ have been calculated by means of the Mathematica package MathTensor [3].
} 
Remark 5.1. The metric (5.11), (5.10) possesses a non-constant scalar curvature $\tau$. Using Mathematica and MathTensor une obtains

$$
\tau:=g^{i k} g^{j l} R_{i j k l}=\frac{-4 h(r)-h^{\prime}(r)^{2}+4 h(r) h^{\prime \prime}(r)}{2 h(r)^{2}},
$$

and the replacement of $h$ by its power series development, determined from (5.11) and (5.10), leads to

$$
\tau=-6-\frac{5}{3} r^{2}-\frac{58}{135} r^{4}-\frac{1213}{11340} r^{6}+O\left(r^{7}\right) .
$$

Consequently, the metric (5.11) is not contained in several classes of Riemannian spaces which require a constant scalar curvature $\tau$. Obviously, (5.11) is not an Einstein space or a space of constant curvature. Furthermore, (5.11) is not a D'Atri space (sce [12: p. 2501); thus the properties of local symmetry and local isotropy are also excluded (see [12: p. 251]). Finally, metric (5.11) can not be locally homogeneous, too.

Remark 5.2. For all dimensions $\operatorname{dim} M>3$ there exist also examples $(M, g)$ of Riemannian manifolds such that the $\left(\partial^{(r)} R\right)_{b}$ have a part in the ideal $\hat{J}_{(r)} \oplus \tilde{J}_{(r)}$. For instance, such an example is given by a product manifold $(M, g)=\left(M^{\prime}, g^{\prime}\right) \times\left(M^{\prime \prime}, g^{\prime \prime}\right)$ which is formed from a 3-dimensional Riemannian manifold $\left(M^{\prime}, g^{\prime}\right)$ according to Proposition 5.2 and a flat Riemannian manifold $\left(M^{\prime \prime}, g^{\prime \prime}\right)$. Let us assume that $\left\{M^{\prime}, x^{\prime}\right\}$ is a normal coordinate system according to Proposition 5.2 with centre $P^{\prime} \in M^{\prime}$. Then we can determine a product chart $x=x^{\prime} \times x^{\prime \prime}$ of $M^{\prime} \times M^{\prime \prime}$ around any point $\left(P^{\prime}, P^{\prime \prime}\right) \in M^{\prime} \times M^{\prime \prime}$ which is a normal coordinate system with respect to $g$. At most the coordinates

$$
R_{\mathbf{i}^{\prime} j^{\prime} k^{\prime} l^{\prime}}\left(x^{a^{\prime}}\right) \quad, \quad a^{\prime}, i^{\prime}, j^{\prime}, k^{\prime}, l^{\prime}=1,2,3 \text {, }
$$

of the curvature tensor do not vanish with respect to $x$. We see from the proof of Proposition 5.2 that the $R_{i^{\prime} j^{\prime} k^{\prime} l^{\prime}}$ contain homogeneous polynomials of orders 2,4 and 6 in $x^{1}, x^{2}, x^{3}$ such that there holds $\left.\partial^{(m)} R\right|_{\left(\mu^{\prime}, p^{\prime \prime}\right)} \neq 0$ for $m=2,4,6$. On the other hand, the expressions $R_{i^{\prime} j k l^{\prime}} x^{j} x^{k}=R_{i^{\prime} j^{\prime} k^{\prime} l^{\prime}} x^{j^{\prime}} x^{k^{\prime}}$ are quadratic polynomials in the coordinates $x^{1}, x^{2}, x^{3}$, and the expressions $R_{i j k l} x^{j} x^{k}$ vanish if $i>3$ or $l>3$. Thus we obtain $\left.\partial^{(m)} \breve{R}\right|_{\left(P^{\prime}, P^{\prime \prime}\right)}=0$ for $m \geq 1$. But then the same arguments which we used in the proof of Proposition 5.2 tell us that $\left(\partial^{(m)} R\right)_{b} \in \hat{J}_{(m)} \oplus \tilde{J}_{(m)}$ for all $b=\left\{v_{1}, \ldots, v_{m+4}\right\} \subset$ $\left(M^{\prime} \times M^{\prime \prime}\right)_{\left(P^{\prime}, P^{\prime \prime}\right)}$ and $m \geq 1$, and that non-vanishing $\left(\partial^{(m)} R\right)_{b}$ exist for at least $m=$ $2,4,6$.

\section{The equality of the tensor algebras $\mathcal{R}$ and $\mathcal{R}^{s}$}

Now we return to the question whether the tensor algebra $\mathcal{R}(1.6)$ is equal to the tensor algebra $\mathcal{R}^{s}$ (1.8). To answer this question, we use the following proposition which follows easily from results of $[6]$.

Proposition 6.1. Let $\nabla_{()}^{(r)} R$ denote the symmetrized covariant derivative of order $r$ of the Riemannian curvature tensor with coordinates $\nabla_{\left(i_{5}\right.} \ldots \nabla_{\left.i_{r+1}\right)} R_{i_{1} \ldots i_{1}}$. Further, us: put $\nabla_{()}^{(0)} R:=R$. Then there holds true for $r \geq 0$

$$
y_{i_{r}}^{*} \nabla_{()}^{(r)} R=\mu_{r} \nabla_{()}^{(r)} R, \ldots \mu_{r}=2(r+3)(r+2) r ! \ldots \ldots
$$

if $y_{\ell_{r}}$ is the Young symometrizer of the standard tablean $t_{r}$ (3.18). 
Proof. We will carry out here those steps of the proof which are not given explicitely in [6].

In the case $r=0$ the assertion follows from Proposition 3.1, (3.9). Thus we can assume $r \geq 1$ in the following.

Definition 6.1. We denote by $\mathcal{T}_{r, \mathcal{B}} V$ the vector space of complex-valued covariant tensors $T$ of order $r+4$ on a vector space $V$ over $\mathbb{C}$ which have the following properties:

1. Every $T \in \mathcal{T}_{r, \mathcal{B}} V$ possesses the symmetry properties of the Riemannian curvature tensor relating to the indices $i_{1}, \ldots, i_{4}$, i.c.

$$
T_{i_{1} i_{2} i_{3} i_{4} i_{5} \ldots i_{r+4}}=-T_{i_{2} i_{1} i_{3} i_{4} i_{5} \ldots i_{r+4}}=-T_{i_{1} i_{2} i_{4} i_{3} i_{5} \ldots i_{r+4}}=T_{i_{3} i_{4} i_{1} i_{2} i_{3} \ldots i_{r+4}} .
$$

2. Every $T \in \mathcal{T}_{r, B} V$ satisfies the first Bianchi identity relating to the indices $i_{2}, i_{3}, i_{4}$ and the second Bianchi identity relating to the indices $i_{3}, i_{4}, i_{5}$, i.e.

$$
T_{i_{1} i_{2} i_{3} i_{4} i_{3} \ldots i_{r+4}}+T_{i_{1} i_{3} i_{4} i_{2} i_{5} \ldots i_{r+4}}+T_{i_{1} i_{4} i_{2} i_{3} i_{3} \ldots i_{r+4}}=0
$$

and

$$
T_{i_{1} i_{2} i_{3} i_{4} i_{5} \ldots i_{r+4}}+T_{i_{1} i_{2} i_{4} i_{5} i_{3} \ldots i_{r+4}}+T_{i_{1} i_{2} i_{5} i_{3} i_{4} \ldots i_{r+4}}=0 .
$$

3. Every $T \in \mathcal{T}_{r, B} V$ is symmetric in $i_{5}, \ldots, i_{r+4}$.

Furthermore, we assume that there is given an order relation $<$ in the set of the $r+4$ index names of a $T \in \mathcal{T}_{r, \mathcal{B}} V$. Let $a<b<c<d<e$ be the 5 smallest index names. Then there is proved in [6: p. 1154]:

Proposition 6.2. Every coordinate $T_{i_{1} \ldots i_{r+4}}$ of a tensor $T \in \mathcal{T}_{r, \mathcal{B}} V$ with an arbitrary arrangement of its index names can be expressed as a linear combination of the following types of coordinates:

$$
\begin{array}{ll}
T_{a b c d e . . .} & \\
T_{a b c i d \ldots} & \text { with } d<i \\
T_{a \text { rebde... }} & \\
T_{a c b i d \ldots} & \\
T_{a i b j c . .} & \text { with } d<i \\
& \text { with } c<i<j .
\end{array}
$$

The dots represent the ordered sequcnce of the remaining index names. The number of these special coordinates is

$$
1+r+1+r+\frac{r(r+1)}{2}=\frac{(r+1)(r+4)}{2} .
$$

Another result of $[6:$ p. 1162$]$ reads:

Proposition 6.3. If $T$ is an arbitrary covariant tensor of order $r+4$ on $V$, then $y_{i_{r}}^{*} T$ lies in $\mathcal{T}_{r, B} V$. 
Let $Q \subset \mathcal{S}_{r+4}$ be the set of all permutations which transform the ordered sequence of the $r+4$ index names of a covariant tensor $T$ of order $r+4$ into the index arrangements given in Proposition 6.2. Then there follows from Proposition 6.2 that every $T \in \mathcal{T}_{r, \boldsymbol{B}} V$ satisfies

$$
\forall p \in \mathcal{S}_{r+4}: \quad p T=\sum_{q \in Q} a_{p q} q T, a_{p q} \in \mathbb{C}
$$

with coefficients $a_{p q}$ which are independent on $T$. Taking into account the relation

$$
\forall b=\left\{v_{1}, \ldots, v_{r+4}\right\} \subset V, \forall p, s \in \mathcal{S}_{r+4}: \quad(s T)_{b}(p)=T_{b}(p \circ s),
$$

which is a consequence of

$$
(s T)_{b}=T_{b} \cdot s^{*}=\sum_{p^{\prime} \in \mathcal{S}_{\mathrm{r}+4}} T_{b}\left(p^{\prime}\right) p^{\prime} \circ s^{-1}=\sum_{p \in \mathcal{S}_{\mathrm{r}+4}} T_{b}(p \circ s) p
$$

we obtain from (6.3)

$$
T_{b}=\sum_{s \in \mathcal{S}_{r+4}}(s T)_{b}(i d) s=\sum_{s \in S_{r+4}} \sum_{q \in Q} a_{s q}(q T)_{b}(i d) s=\sum_{q \in Q} T_{b}(q) u_{q}
$$

where $u_{q}:=\sum_{s \in S_{r+4}} a_{s q} s$.

Now, let $W_{\mathcal{B}}(V):=\mathcal{L}\left\{T_{b} \mid T \in \mathcal{T}_{r, \mathcal{B}} V, b=\left\{v_{1}, \ldots, v_{r+4}\right\} \subset V\right\}$ be the vector subspace of $\mathbb{C}\left[\mathcal{S}_{r+4}\right]$ generated by all $T_{b}$ of the tensors $T \in \mathcal{T}_{r, \mathcal{B}} V$. Then equation (6.4) yields $W_{\mathcal{B}}(V) \subseteq \mathcal{L}\left\{u_{q} \mid q \in Q\right\}$ and $\operatorname{dim} W_{\mathcal{B}}(V) \leq|Q|=(r+4)(r+1) / 2$.

Proposition 6.3 means that $\left(y_{t_{\tau}}^{*} T\right)_{b} \in W_{\mathcal{B}}(\bar{V})$ for all subsets $b=\left\{v_{1}, \ldots, v_{r+4}\right\} \subset$ $V$. In the following we assume $\operatorname{dim} V \geq r+4$. Then there exists a vector set $b_{0}=$ $\left\{v_{1}, \ldots, v_{r+4}\right\} \subset V$ such that $\mathbb{C}\left[\mathcal{S}_{r+4}\right]$ is generated by the $T_{b_{0}}$ of all covariant tensors $T$ of order $r+4$ (sec [5: Lemma 2.1]) and consequently the left ideal $J_{(r)}=\mathbb{C}\left[\mathcal{S}_{r+4}\right] \cdot y_{\iota_{r}}$ is spanned by the $T_{b_{0}} \cdot y_{t_{r}}=\left(y_{i_{r}}^{*} T\right)_{b_{0}}$ of all covariant tensors $T$ of order $r+4$. Thus we obtain $J_{(r)} \subseteq W_{B}(V)$. But since $\operatorname{dim} J_{(r)}=(r+4)(r+1) / 2$ because of $(4.7)$, there follows $J_{(r)}=\bar{W}_{\mathcal{B}}(V)$.

In the case $m:=\operatorname{dim} V<r+4$ we introduce an $(r+4)$-dimensional vector space $\tilde{V}$ which we map linearly onto $V$ by means of a linear mapping $\phi: \tilde{V} \rightarrow V$ defined on given bases $\left\{u_{1}, \ldots, u_{m}\right\}$ of $V$ and $\left\{\tilde{u}_{1}, \ldots, \tilde{u}_{r+4}\right\}$ of $\tilde{V}$ by the rulc

$$
\phi\left(\tilde{u}_{i}\right):=\left\{\begin{array}{ll}
u_{i} & \text { if } i=1, \ldots, r n \\
0 & \text { if } i=r n+1, \ldots, r+4 .
\end{array} .\right.
$$

Then the pull back. $\left(\phi^{*} T\right)\left(\tilde{v}_{1}, \ldots, \tilde{v}_{r+4}\right):=T\left(\phi\left(\tilde{v}_{1}\right), \ldots, \phi\left(\tilde{v}_{r+4}\right)\right), \tilde{v}_{i} \in \tilde{V}$, of every tensor $T \in \mathcal{T}_{r, B} V$ lies in $\mathcal{T}_{r, B} \tilde{V}$. Every vector set $b=\left\{v_{1}, \ldots, v_{r+4}\right\} \subset V$ corresponds to a uniquely determined vector set $\tilde{b}=\left\{\tilde{v}_{1}, \ldots, \tilde{v}_{r+4}\right\} \subset \mathcal{L}\left\{\tilde{u}_{1}, \ldots, \tilde{u}_{m}\right\}$ via $v_{i}=\phi\left(\tilde{v}_{i}\right)$. Thus there holds true $T_{b}=\left(\phi^{*} T\right)_{\tilde{b}} \in W_{\mathcal{B}}(\tilde{V})=J_{(r)}$ for cvery $T \in \mathcal{T}_{r, \mathcal{B}} V, b \subset V$.

Let now $V=M_{P}$, be a tangent space of our differentiable manifold $M$ in a point $P \in M$. Then there is $\nabla_{()}^{(r)} R \in \mathcal{T}_{r, \mathcal{B}} M_{P}$. This leads to $\left(\nabla_{()}^{(r)} R\right)_{b} \in J_{(r)}=\mathbb{C}\left[\mathcal{S}_{r+4}\right] \cdot y_{\iota_{r}}$, that means $\left(\nabla_{()}^{(r)} R\right)_{b}=x \cdot y_{\ell_{r}}$ with some $x \in \mathbb{C}\left[\mathcal{S}_{r+4}\right]$. Now taking into account (4.16) and (4.14) we obtain

$$
\left(y_{t_{r}}^{*} \nabla_{()}^{(r)} R\right)_{b}=x \cdot y_{t_{r}} \cdot y_{t_{r}}=\mu_{r}\left(\nabla_{0}^{(r)} R\right)_{b}
$$

for every vector set $b=\left\{v_{1}, \ldots, v_{r+1}\right\} \subset M_{p}$ by which Proposition 6.1 is proved 
Now the version of Proposition 3.1 for $\nabla_{()}^{(r)} R$ reads

Corollary 6.1. Let $r \geq 0$. Then the group ring elernent $\left(\nabla_{()}^{(r)} R\right)_{b} \in \mathbb{C}\left[\mathcal{S}_{r+4}\right]$ is contained in the left ideal

$$
J_{(r)}=\mathbb{C}\left[\mathcal{S}_{r+4}\right] \cdot y_{t_{r}}
$$

of $\mathbb{C}\left[\mathcal{S}_{r+4}\right]$ for every set of vectors $b=\left\{v_{1}, \ldots, v_{r+4}\right\} \subset M_{P}, P \in M$.

Since $J_{(r)}$ is minimal, the problem of decomposition of $J_{(r)}$ does not arise.

Theorem 6.1. We denote by $\nabla^{(r)} \breve{R}$ the 'stronger' symmetrized covariant derivative of the Riemannian curvature tensor of order $r$ the coordinates of which have the form

$$
\begin{gathered}
\left(\nabla^{(r)} \breve{R}\right)_{i_{1} i_{2} i_{3} i_{4} i_{5} \ldots i_{r+4}}:=\nabla_{\left(i_{5}\right.} \ldots \nabla_{i_{r+4}} R_{\left.\left|i_{1}\right| i_{2} i_{3}\right) i_{4}} \\
\left(\nabla^{(0)} \breve{R}\right)_{i_{1} \ldots i_{4}}:=\breve{R}_{i_{1} \ldots i_{4}}:=R_{i_{1}\left(i_{2} i_{3}\right) i_{4}}
\end{gathered}
$$

Then there holds true for $r \geq 0$

$$
\begin{aligned}
& 2 \frac{r+3}{r+1}\left(\nabla_{()}^{(r)} R\right)_{i_{1} i_{2} i_{3} i_{4} i_{5} \ldots i_{r+4}}= \\
& +\left(\nabla^{(r)} \breve{R}\right)_{i_{1} i_{2} i_{3} i_{4} i_{5} \ldots i_{r+4}}-\left(\nabla^{(r)} \breve{R}\right)_{i_{2} i_{1} i_{3} i_{4} i_{5} \ldots i_{r+4}}-\left(\nabla^{(r)} \breve{R}\right)_{i_{1} i_{2} i_{4} i_{3} i_{5} \ldots i_{r+4}} \\
& +\left(\nabla^{(r)} \breve{R}\right)_{i_{4} i_{2} i_{3} i_{1} i_{5} \ldots i_{r+4}}+\left(\nabla^{(r)} \breve{R}\right)_{i_{2} i_{1} i_{4} i_{3} i_{5} \ldots i_{r+4}}-\left(\nabla^{(r)} \breve{R}\right)_{i_{4} i_{1} i_{3} i_{2} i_{5} \ldots i_{r+4}} \\
& -\left(\nabla^{(r)} \breve{R}\right)_{i_{3} i_{2} i_{4} i_{1} i_{5} \ldots i_{r+4}}+\left(\nabla^{(r)} \breve{R}\right)_{i_{3} i_{1} i_{4} i_{2} i_{5} \ldots i_{r+4}} .
\end{aligned}
$$

As a consequence of $(6.5)$, we obtain $\mathcal{R}=\mathcal{R}^{s}$.

Proof. For every subset $b=\left\{v_{1}, \ldots, v_{r+4}\right\} \subset M_{P}$ of the tangent space in an arbitrary point $P \in M$ there holds truc

$$
\left(y_{t_{r}}^{*}\left(\nabla_{()}^{(r)} R\right)\right)_{b}=\mu_{r}\left(\nabla_{0}^{(r)} R\right)_{b} \text { and }\left(\nabla^{(r)} \breve{R}\right)_{b}=\frac{1}{(r+2) !}\left(\epsilon^{*}\left(\nabla_{0}^{(r)} R\right)\right)_{b} .
$$

Then using (4.14) we can write

$$
\begin{aligned}
\mu_{r}\left(\nabla_{()}^{(r)} R\right)_{b} & =\left(y_{t_{r}}^{*}\left(\nabla_{()}^{(r)} R\right)\right)_{b}=\left(\nabla_{()}^{(r)} R\right)_{b} \cdot y_{t_{r}}=-\frac{1}{\mu_{r}}\left(\nabla_{()}^{(r)} R\right)_{b} \cdot y_{t_{r}} \cdot \epsilon \cdot \eta \\
& =-\frac{1}{\mu_{r}}\left(\eta^{*} \cdot \epsilon^{*} \cdot y_{t_{r}}^{*}\left(\nabla_{()}^{(r)} R\right)_{b}=-\left(\eta^{*} \cdot \epsilon^{*}\left(\nabla_{()}^{(r)} R\right)\right)_{b}\right. \\
& =-(r+2) !\left(\eta^{*}\left(\nabla^{(r)} \breve{R}\right)\right)_{b} .
\end{aligned}
$$

Now equation (6.5) can be: proved by the same arguments which we applied to show (4.15)

Acknowledgements. The investigations of this paper originated from a suggestion of Professor P. Gïnther. I an greatly indebted to him for the constant support and scientific guidance which he has given me during my whole professional development.

I wish to express nny sincerest thanks to Prof. G. Eisenreich for checks of my work and valuable hints. 


\section{References}

[1] Boerner, H.: Darstellungen von Gruppen (Die Grundlehren der mathematischen Wissenschaften in Einzeldarstellungen: Vol. 74). Berlin - Göttingen - Heidelberg: SpringerVerlag, 1955.

[2] Boerner, H.: Representations of Groups (2. revised Ed.). Amsterdam: North-Holland Publ. Comp., 1970.

[3] Christensen, S. and L. Parker: MathTensor: A System for Doing Tensor Analysis by Computer. Reading (Mass.) et al.: Addison-Wesley, 1994.

[4] Fiedler, B.: PERMS 2.1. Leipzig: Mathematisches Institut, Universität Leipzig, 1997. Will be sended in to MathSource, Wolfram Research Inc.

[5] Fiedler, B.: A use of ideal decomposition in the computer algebra of tensor expressions. Z. Anal. Anw. 16 (1997), $145-164$.

[6] Fulling, S., King, R., Wybourne, B. and C. Cummins: Normal forms for tensor polynomials: I. The Riemann tensor. Class. Quantum Grav. 9 (1992), 1151 - 1197.

[7] Günther, P.: Spinorkalkül und Normalkoordinaten. ZAMM 55 (1975), $203-210$.

[8] Günther, P.: Huygens' Principle and Hyperbolic Equations (Perspectives in Mathematics: Vol. 5). Boston et al.: Academic Press, Inc., 1988.

[9] Herglotz, G.: Über die Bestimmung eines Linienelements in Normalkoordinaten aus dern Riemannschen Krümmungstensor. Math. Annalen 93 (1925), 46 - 53.

[10] James, G. D. and A. Kerber: The Representation Theory of the Symmetric Group (Encyclopedia of Mathernatics and its Applications: Vol. 16). Reading (Mass.) et al.: AddisonWesley Publ. Comp., 1981.

[11] Kerber, A.: Representations of Permutation Groups (Lecture Notes in Mathematics: Vol. 240, 495). Berlin - Heidelberg - New York: Springer-Verlag, 1971, 1975.

[12] Kowalski, O., Prüfer, F. and L. Vanhecke: D'Atri spaces. In: Topics in Geometry: In Memory of Joseph D'Atri. Ed.: Gindikin, S. Boston - Basel - Berlin: Birkhäuser, 1996, pp. 241 - 284. Reprint from: Progress in Nonlinear Differential Equations, Volume 20.

[13] Littlewood, D.: The Theory of Group Characters and Matrix Representations of Groups (2. Ed.). Oxford: Clarendon Press, 1950.

[14] Macdonald, I.: Symmetric Functions and Hall Polynomials. Oxford: Clarendon Press, 1979.

[15] Naimark, M. and A. Stern: Theory of Group Representations (Grundlehren der Mathematischen Wissenschaften: Vol. 246). Berlin - Heidelberg - New York: Springer-Verlag, 1982.

[16] van der Waerden, B.: Algebra. 9., 6. Ed., Vol. I, II. Berlin et al.: Springer-Verlag, 1993.

[17] Weyl, H.: The Classical Groups, their Invariants and Representations. Princeton (New Jersey): Princeton University Press, 1939.

[18] Wolfram, S.: Mathematica (2. Ed.). Bonn et al.: Addison-Wesley, 1992. In German.

Received 19.6.1997. 